Supporting Information for

\title{
Synthesis and Characterization of Ru Cubic Nanocages with a Face- Centered-Cubic Structure by Templating with Pd Nanocubes
}

Ming Zhao,$^{\dagger}$ Legna Figueroa-Cosme,$^{\dagger}$ Ahmed O. Elnabawy, ${ }^{\S}$ Madeline Vara, ${ }^{\dagger}$ Xuan Yang, ${ }^{\dagger}$ Luke T. Roling, ${ }^{\S}$ Miaofang Chi, ${ }^{\#}$ Manos Mavrikakis, ${ }^{*} \S$ and Younan Xia ${ }^{*,+,},+$

${ }^{\dagger}$ School of Chemistry and Biochemistry, Georgia Institute of Technology, Atlanta, Georgia 30332, United States

${ }^{\S}$ Department of Chemical \& Biological Engineering, University of Wisconsin-Madison, Madison, Wisconsin 53706, United States

ॠThe Wallace H. Coulter Department of Biomedical Engineering, Georgia Institute of Technology and Emory University, Atlanta, Georgia 30332, United States

${ }^{\#}$ Center for Nanophase Materials Sciences, Oak Ridge National Laboratory, Oak Ridge, Tennessee 37831, United States

*E-mail: manos@engr.wisc.edu for computational work

"E-mail: younan.xia@bme.gatech.edu for experimental work. 


\section{Chemicals and Materials.}

Ethylene glycol (EG, 99\%) was obtained from J. T. Baker. Sodium tetrachloropalladate(II) $\left(\mathrm{Na}_{2} \mathrm{PdCl}_{4}, \quad 99.99 \%\right)$, ruthenium(III) chloride hydrate $\left(\mathrm{RuCl}_{3} \cdot \mathrm{xH}_{2} \mathrm{O}, \quad 99.99 \%\right)$, poly(vinylpyrrolidone) (PVP, MW $\approx 55000$ ), L-ascorbic acid (AA, 99\%), potassium bromide $(\mathrm{KBr}, 99 \%)$, potassium chloride $(\mathrm{KCl}, 99 \%)$, hydrochloric acid $(\mathrm{HCl}, 37 \%)$, and iron(III) chloride $\left(\mathrm{FeCl}_{3}, 97 \%\right)$ were all obtained from Sigma-Aldrich and used as received. All aqueous solutions were prepared using deionized (DI) water with a resistivity of $18.2 \mathrm{M} \Omega \mathrm{cm}$.

\section{Synthesis of 6-nm, 10-nm, and 18-nm Pd Nanocubes.}

Pd nanocubes $(6,10$, and $18 \mathrm{~nm}$ in edge length) were synthesized using protocols reported by our group. ${ }^{1}$ For the synthesis of 10-nm Pd cubes, PVP (105 mg), AA (60 mg), and KBr (300 $\mathrm{mg}$ ) were dissolved in DI water $(8.0 \mathrm{~mL})$, and then placed in a vial and pre-heated at $80{ }^{\circ} \mathrm{C}$ in an oil bath under magnetic stirring for $10 \mathrm{~min}$. Subsequently, $3 \mathrm{~mL}$ of aqueous $\mathrm{Na}_{2} \mathrm{PdCl}_{4}$ solution (19 $\left.\mathrm{mg} \mathrm{mL}^{-1}\right)$ was injected into the preheated solution in one shot. The reaction solution was maintained at $80{ }^{\circ} \mathrm{C}$ under magnetic stirring for $3 \mathrm{~h}$. The product was collected by centrifugations, washed three times with DI water to remove excess PVP and inorganic ions, and then re-dispersed in $6 \mathrm{~mL}$ EG. For the syntheses of $6 \mathrm{~nm}$ and $18 \mathrm{~nm}$ nanocubes, the procedure was kept the same except that $\mathrm{KBr}(300 \mathrm{mg})$ was replaced by $\mathrm{KBr}(5 \mathrm{mg})$ and $\mathrm{KCl}$ (185 mg), and $\mathrm{KBr}(600 \mathrm{mg})$, respectively.

\section{Synthesis of Pd@Ru Core-shell Nanocubes.}

In a standard synthesis of $\mathrm{Pd} @ \mathrm{Ru}$ core-shell nanocrystals, $50 \mathrm{mg}$ AA and $100 \mathrm{mg}$ PVP were dissolved in $8 \mathrm{~mL}$ EG. The resulting solution was preheated at $200{ }^{\circ} \mathrm{C}$ under magnetic stirring for $10 \mathrm{~min}$ in a three-necked flask. Then, $1 \mathrm{~mL}$ of a suspension of $10-\mathrm{nm} \mathrm{Pd}$ cubes $(2$ $\mathrm{mg} \mathrm{mL} \mathrm{m}^{-1}$ ) was added into the flask and kept at $200{ }^{\circ} \mathrm{C}$ under magnetic stirring for 1 hour. Subsequently, $2 \mathrm{mg} \mathrm{RuCl}{ }_{3} \cdot \mathrm{xH}_{2} \mathrm{O}$ and $250 \mathrm{mg}$ PVP were dissolved in $20 \mathrm{~mL}$ EG and injected to the flask at a rate of $1 \mathrm{~mL} \mathrm{~h}^{-1}$ using a syringe pump. The reaction was allowed to continue for an additional $10 \mathrm{~min}$ to complete. The resultant product was collected by centrifugation, washed once with acetone and twice with water, and then re-dispersed in $8 \mathrm{~mL} \mathrm{DI}$ water. The procedure for the syntheses of 6 and $18 \mathrm{~nm} \mathrm{Pd@Ru} \mathrm{nanocubes} \mathrm{was} \mathrm{similar} \mathrm{to} \mathrm{that} \mathrm{of} 10 \mathrm{~nm}$ $\mathrm{Pd} @ \mathrm{Ru}$ nanocubes. $2 \mathrm{mg}$ of 6-nm and 18-nm Pd nanocubes were used as seeds respectively and $3.64 \mathrm{mg}$ and $1.04 \mathrm{mg} \mathrm{RuCl} 3 \cdot \mathrm{xH}_{2} \mathrm{O}$ was injected accordingly. The number of grown $\mathrm{Ru}$ layers was the same for these three kinds of Pd seeds. All other synthetic procedures were kept 
the same as for the 10-nm Pd@Ru cube synthesis.

\section{Formation of Ru Nanocages.}

Chemical etching was conducted in an acidic aqueous solution to generate Ru nanocages. Typically, $\mathrm{KBr}$ (300 mg), PVP (50 mg), $\mathrm{FeCl}_{3}$ (50 mg), $\mathrm{HCl}$ (0.3 mL, 37\%), DI water (5.7 mL), and $1 \mathrm{~mL}$ as-prepared $\mathrm{Pd} @ \mathrm{Ru}$ core-shell nanocrystals were mixed together in an 18-mL vial. The mixture was transferred into an oil bath heated at $100{ }^{\circ} \mathrm{C}$ under magnetic stirring for $3 \mathrm{~h}$. After that, the product was collected by centrifugation, washed three times with ethanol, and then re-dispersed in DI water for further characterization.

\section{Morphological, Structural, and Elemental Analysis.}

Transmission electron microscopy (TEM) was conducted with an HT7700 microscope operated at $120 \mathrm{kV}$. High-resolution transmission electron microscopy (HRTEM) images were acquired on a Cs-corrected FEI Titan 80/300 kV TEM/STEM microscope at Oak Ridge National Laboratories (ORNL). All high-angle annular dark field scanning transmission electron microscopy (HAADF-STEM) images and energy-dispersive X-ray spectroscopy (EDX) data were acquired using an aberration-corrected Hitachi HD-2700 200 kV STEM equipped with a Brüker Quantax 400/S - STEM EDX detector at Georgia Institute of Technology. Inductively coupled plasma mass spectrometry (ICP-MS, NexION 300Q, PerkinElmer) was used for a quantitative analysis of metal content in the samples. X-ray diffraction (XRD) patterns were obtained with a PANalytical X'Pert PRO Alpha-1 diffractometer using $1.8 \mathrm{~kW}$ Ceramic Copper tube source.

\section{DFT Calculations.}

Periodic, self-consistent density functional theory (DFT) calculations were performed using the Vienna ab initio Simulation Package (VASP) code. ${ }^{2,3}$ Projector augmented wave (PAW) potentials ${ }^{4,5}$ were used to describe electron-ion interactions, and the generalized gradient approximation (GGA-PW91) was used to define the exchange-correlation functional. ${ }^{6}$ Our calculations expand the wave function in a basis set of plane waves, truncated at a kinetic energy cutoff of $400 \mathrm{eV}$. All calculations were performed for adsorption on a single side of a metal slab, periodically repeated in a $2 \times 2$ unit cell, corresponding to $1 / 4$ monolayer (ML) coverage (except for studies of Ru surface diffusion and substitution, where both $2 \times 2$ and $3 \times 3$ 
unit cells were used as explained below). At least $13 \AA$ of vacuum were used to separate periodic images in the $z$-direction, to avoid interactions between images. The first Brillouin zone was sampled using a $(6 \times 6 \times 1)$ Monkhorst-Pack $k$-point mesh. ${ }^{7}$ Geometric optimization was performed until the Hellmann-Feynman forces on all atoms converged to less than 0.01 eV/Å. We calculated the binding energies (BE) of surface adsorbates according to:

$$
\mathrm{BE}=\mathrm{E}_{\text {total }}-\mathrm{E}_{\text {slab }}-\mathrm{E}_{\text {gas-phase adsorbate }}
$$

where $E_{\text {total }}$ is the total energy of the metal slab with the adsorbate, $E_{\text {slab }}$ is the total energy of the slab excluding the adsorbate, and $E_{\text {gas-phase adsorbate }}$ is the energy of the adsorbate in the gas phase at infinite separation from the slab. Activation energy barriers for bond dissociation steps and diffusion/substitution events were calculated using the climbing image nudged elastic band (CI-NEB) method, $^{8}$ in which initial and final states were connected with seven interpolated images that were each converged to forces less than $0.1 \mathrm{eV} / \AA$. Vibrational frequency calculations were performed to confirm a single imaginary vibrational mode for the calculated transition states. The vibrational frequencies of translational, vibrational, and rotational modes assumed a quantum harmonic oscillator, and were estimated by second-order numerical differentiation of forces, using a step size of $0.015 \AA$.

Surface diffusion and substitution calculations were performed for Ru adatoms on a fourlayer Pd(100) slab model, with the top two layers relaxed and the bottom two fixed at their bulk positions. The optimized lattice constant of Pd was calculated to be $3.958 \AA$, in good agreement with the experimental value of $3.89 \AA . .^{9}$ In addition to performing calculations in a $2 \times 2$ unit cell, we also performed these diffusion and substitution calculations in a $3 \times 3$ unit cell, corresponding to $1 / 9 \mathrm{ML}$ coverage, to gauge the effect of surface coverage on the calculated energetics. All parameters for the $3 \times 3$ unit cell were kept the same as those for the $2 \times 2$ unit cell (including 6×6×1 k-point mesh).

We performed calculations on six model surfaces to evaluate variations in catalytic activity resulting from the substantial structural differences between the close-packed $h c p$ (0001) Ru structure and the as-synthesized $f c c-(100) \mathrm{Ru}$ nanocages. We calculated the lattice parameters of bulk $h c p \mathrm{Ru}$ to be $\mathrm{a}=2.729 \AA$ and $\mathrm{c} / \mathrm{a}=1.577$, in close agreement with the experimental values of $\mathrm{a}=2.706 \AA$ and $\mathrm{c} / \mathrm{a}=1.582 .{ }^{9}$ These parameters were used to construct the $h c p$-(0001) slab model, which is used as a reference state for traditional hcp Ru catalysts. We 
also calculated the lattice constant of $\mathrm{Ru}$ in the bulk $f c c$ crystal structure to be $3.821 \AA$, from which we constructed two slab models to gauge the effect of changing the structure of hcp(0001) Ru to close-packed $f c c-(111)$ and undercoordinated $f c c-(100)$ surfaces. All three of these slab models utilized four-layer slabs with the bottom two layers fixed at their bulk positions, while the top two layers (and adsorbates) were allowed to fully relax.

Since the as-synthesized Ru catalysts are hollow, free-standing structures, we constructed additional models in a similar fashion to that we used recently for modeling Pt-Pd nanocages. ${ }^{10}$ In particular, we modeled the as-synthesized six-layer nanocages as a six-layer membrane with a (100)-like surface arrangement of atoms and all atoms relaxed. The interatomic distance within layers was optimized; for the model consisting of pure Ru (i.e., the $f c c-(100)$-cage), the interatomic distance within each layer was $2.685 \AA$. We note that these optimized interatomic distances were slightly constricted relative to the $f c c-(100)$ slab, which possesses an interatomic distance of $2.702 \AA$. Finally, in order to model the synthesized structures most accurately, we exchanged two $\mathrm{Ru}$ atoms in the cage model with $\mathrm{Pd}$ atoms, yielding a mass ratio of $8.7 \% \mathrm{Pd}$, consistent with the $8.8 \% \mathrm{Pd}$ in the nanocages characterized by ICP-MS. The optimized interatomic distance for this structure is $2.690 \AA$. As in our previous work, ${ }^{10}$ we assumed that all $\mathrm{Pd}$ in contact with the etching agent should be removed during the etching process, so we therefore included no Pd in the exposed top or bottom layers of the membrane. We instead chose to disperse the two Pd atoms in the slab, placing one in the second membrane layer from the top (denoted cage-(100) $\mathrm{Pd} 2$ ) and one in the fourth membrane layer from the top (i.e. third layer from the bottom, denoted cage-(100) $\mathrm{Pd} 3)$. We note that this arrangement of $\mathrm{Pd}$ atoms in the membrane structure yields two different surface profiles in our membrane model (one with $\mathrm{Pd}$ in the second layer from the surface, and the other with Pd in the third layer from the surface). To elucidate the effect of $\mathrm{Pd}$ position within the slab, we therefore performed calculations on both exposed surfaces (one at a time) of the membrane. We expect that the $\mathrm{Pd}$ is dispersed somewhat randomly in the interior of the true nanocage structures due to the intermixing of $\mathrm{Pd}$ and $\mathrm{Ru}$ during the synthesis of the core-shell particles. We therefore do not attempt to determine the minimum energy position of $\mathrm{Pd}$, but rather investigate this effect of placing $\mathrm{Pd}$ in different positions relative to the surface to elucidate the effect of different types of adsorption sites (and the resulting differences in energetics) in the true catalyst. 
As a final note, we observe that although $4 \mathrm{~d}$ transition metals - to which Ru belongs - do not show magnetism in their natural bulk crystal structures, changes to those crystal structures could impart ferromagnetism. ${ }^{11}$ In fact, different phases of $\mathrm{Ru}$ have been studied computationally for the sole purpose of investigating their magnetic properties. ${ }^{12}$ We have performed test spin-polarized calculations with and without adsorbates on all model systems described above, and calculated negligible magnetic moments and negligible differences in total energies when compared to corresponding spin-restricted calculations (consistent with the previous computational work for $f c c \mathrm{Ru}) .{ }^{12}$ As such, all results presented in this work were calculated without spin-polarization. 


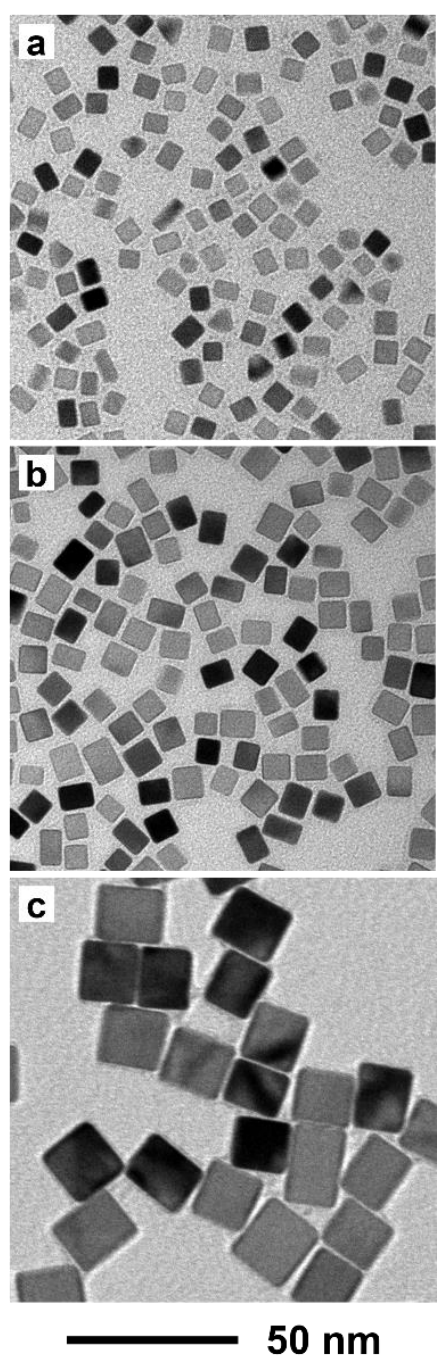

Figure S1. TEM images of Pd seeds with different edge lengths. (a) $6 \mathrm{~nm}$, (b) $10 \mathrm{~nm}$, and (c) $18 \mathrm{~nm}$. 


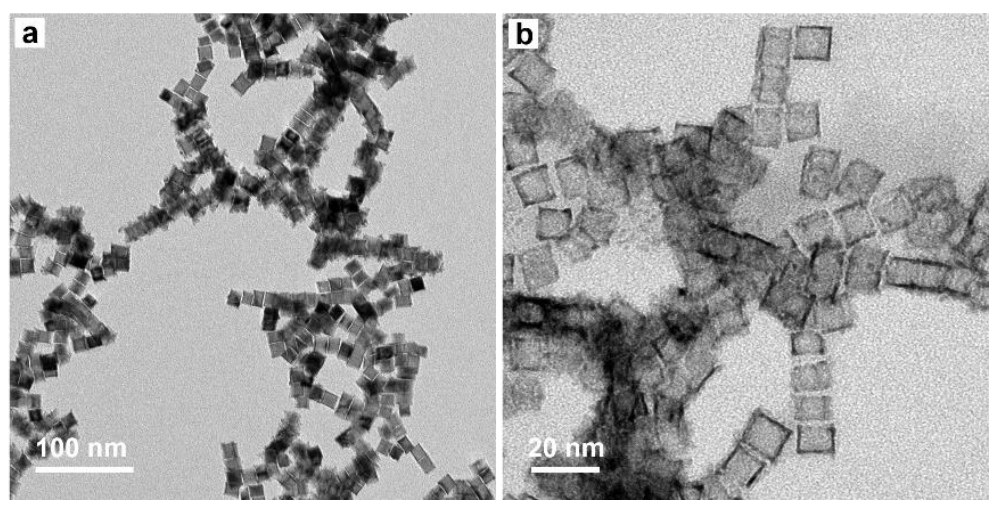

Figure S2. TEM images of (a) typical Pd@Ru nanocrystals prepared from 10-nm Pd cubes using the standard procedure without adding PVP into the Ru precursor solution, (b) Ru nanocages produced after chemical etching of (a). 


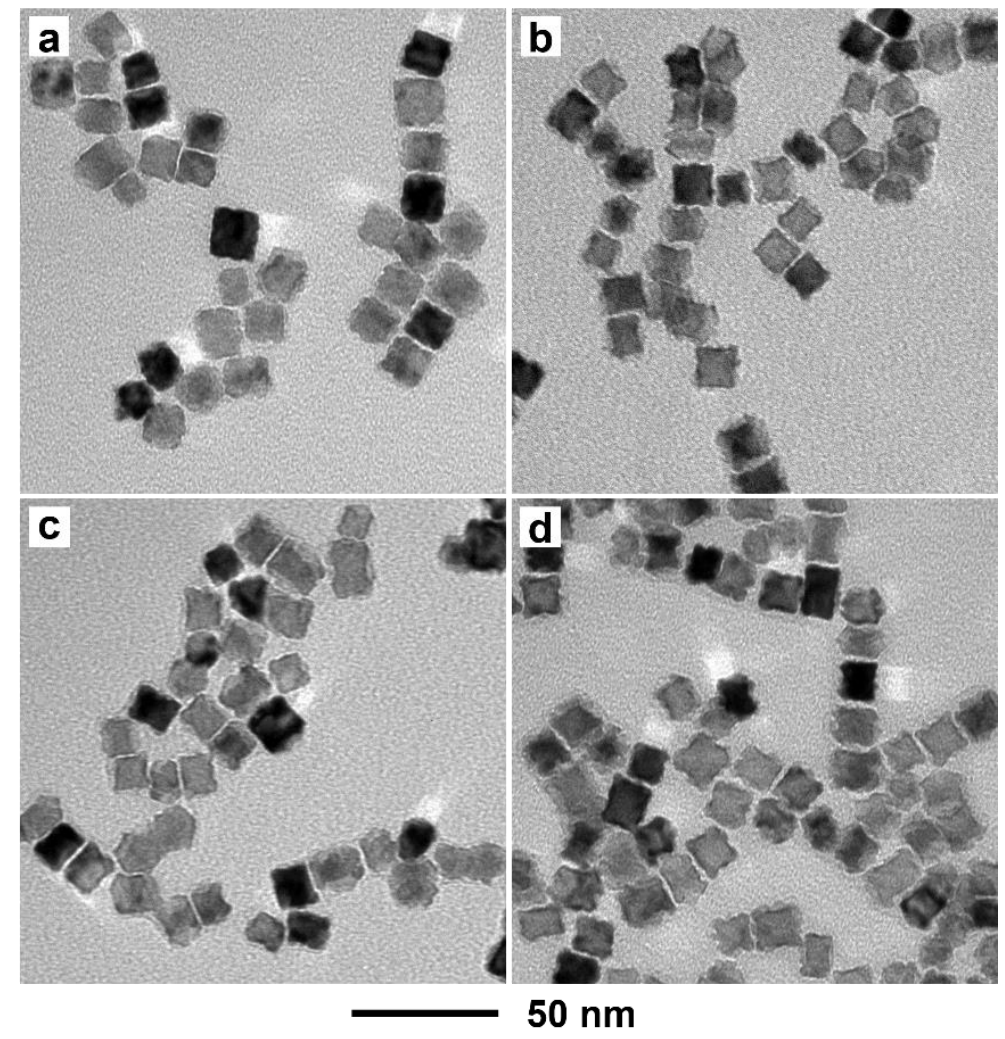

Figure S3. TEM images of typical Pd@Ru nanocubes prepared from 10-nm Pd cubes using the standard procedure except with different injection rates. (a) $2 \mathrm{~mL} \mathrm{~h}^{-1}$, (b) $5 \mathrm{~mL} \mathrm{~h}^{-1}$, (c) 10 $\mathrm{mL} \mathrm{h}^{-1}$, and (d) $20 \mathrm{~mL} \mathrm{~h}^{-1}$. 
Table S1. Comparison of elemental composition of $\mathrm{Pd} @ \mathrm{Ru}$ nanocubes prepared by the standard procedure except at different temperature.

\begin{tabular}{c|c|c}
\hline Reaction Temperature & Percentage of Pd $(\mathrm{wt} \%)$ & Percentage of Ru $(\mathrm{wt} \%)$ \\
\hline $175^{\circ} \mathrm{C}$ & 70.9 & 29.1 \\
\hline $150^{\circ} \mathrm{C}$ & 72.2 & 27.8 \\
\hline $125^{\circ} \mathrm{C}$ & 73.3 & 26.7 \\
\hline $100^{\circ} \mathrm{C}$ & 74.0 & 26.0 \\
\hline
\end{tabular}




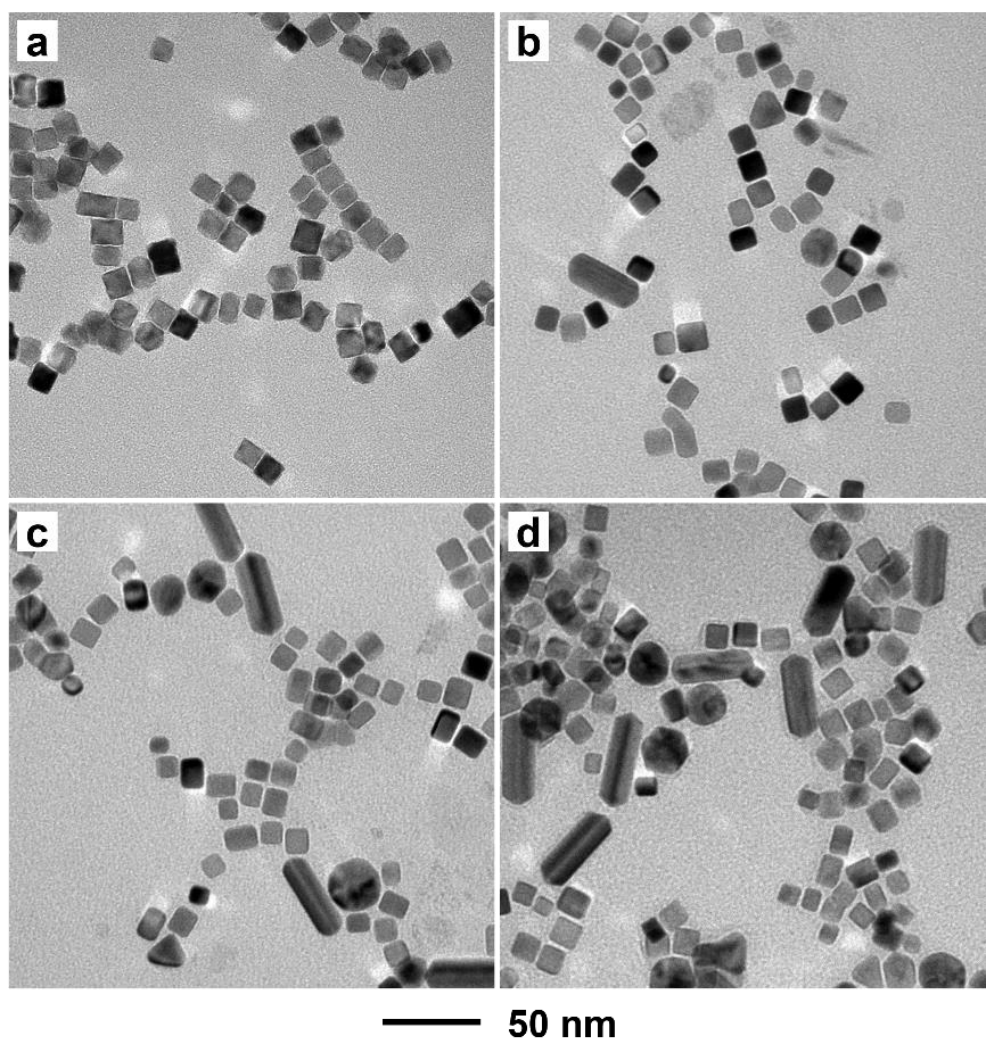

Figure S4. TEM images of typical Pd@Ru core--shell nanocubes prepared from 10-nm Pd cubes using the standard procedure except at different reaction temperatures. (a) $175{ }^{\circ} \mathrm{C}$, (b) $150{ }^{\circ} \mathrm{C}$, (c) $125^{\circ} \mathrm{C}$, and (d) $100^{\circ} \mathrm{C}$. 
Table S2. Comparison of elemental composition of $\mathrm{Pd} @$ Ru nanocubes and nanocages prepared using the standard procedure.

\begin{tabular}{c|c|c}
\hline & Percentage of Pd $(\mathrm{wt} \%)$ & Percentage of $\mathrm{Ru}(\mathrm{wt} \%)$ \\
\hline Before etching & 68.6 & 31.4 \\
\hline After etching & 8.8 & 91.2 \\
\hline
\end{tabular}


Table S3. Calculated activation energies for "hopping" diffusion and substitution of $\mathrm{Ru}$ adatoms on $\operatorname{Pd}(100)$. The energy of substitution (i.e., $\Delta \mathrm{E}$ ) is provided in parentheses; hopping is thermoneutral. All values are in $\mathrm{eV}$.

\begin{tabular}{c|c|c}
\hline Unit Cell & Hopping Barrier & Substitution Barrier \\
\hline $2 \times 2$ & 1.11 & $0.84(-0.66)$ \\
\hline $3 \times 3$ & 1.11 & $0.57(-0.66)$ \\
\hline
\end{tabular}




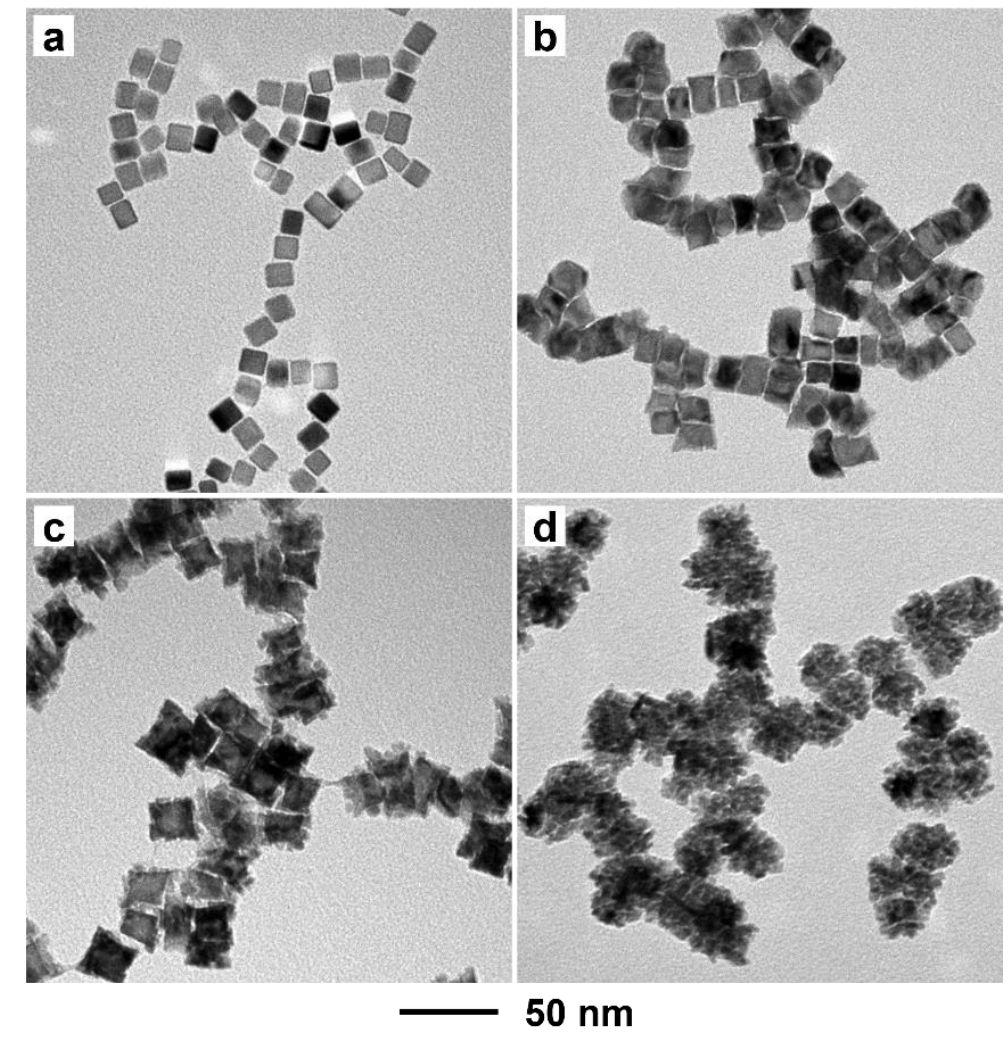

Figure S5. TEM images of typical Pd@Ru nanocrystals prepared from 10-nm Pd cubes using the standard procedure except with different amounts of Ru precursor while keeping the overall volume $20 \mathrm{~mL}$. (a) $1 \mathrm{mg}$, (b) $4 \mathrm{mg}$, (c) $10 \mathrm{mg}$, and (d) $20 \mathrm{mg}$. 


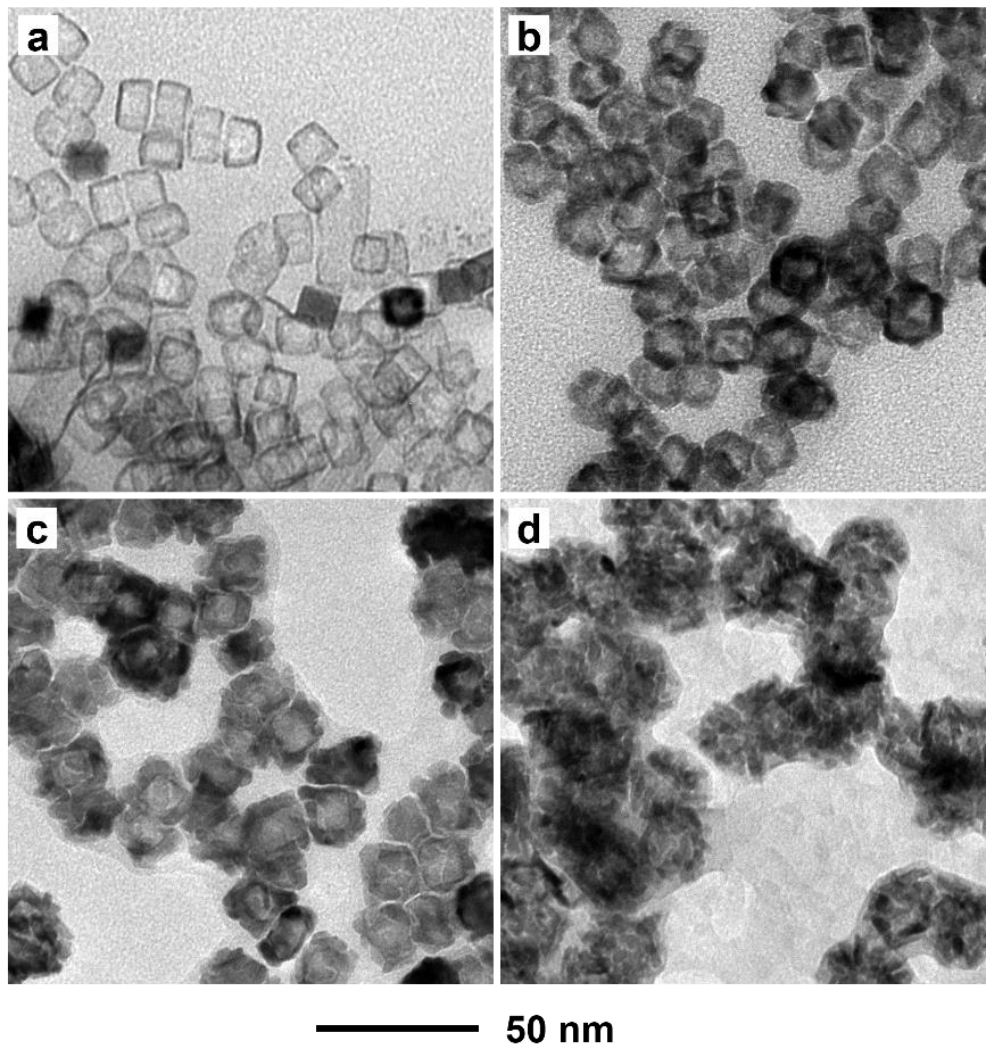

Figure S6. Typical TEM images of Pd@Ru nanocages prepared from 10-nm Pd cubes using the standard procedure except employing different amounts of Ru precursor while keeping the overall volume $20 \mathrm{~mL}$. (a) $1 \mathrm{mg}$, (b) $4 \mathrm{mg}$, (c) $10 \mathrm{mg}$, and (d) $20 \mathrm{mg}$. 
Table S4. Binding energies (in eV) of 14 atomic and molecular species on all six model surfaces studied. Calculated values on surfaces to which each species binds most strongly are shown in bold. Reference energy is adsorbate in the gas phase, at infinite separation from the model surface.

\begin{tabular}{|c|c|c|c|c|c|c|}
\hline Species & $h c p-(0001)$ & $|f c c-(111)|$ & $f c c-(100)$ & cage-(100) & cage- $(100)_{\mathrm{Pd} 2}$ & cage $-(100)_{\mathrm{Pd} 3}$ \\
\hline $\mathrm{C}$ & -7.55 & -7.76 & -8.42 & -8.44 & -8.55 & -8.34 \\
\hline $\mathrm{N}$ & -6.00 & -6.31 & -6.25 & -6.30 & -6.44 & -6.27 \\
\hline $\mathrm{O}$ & -6.00 & -6.25 & -6.00 & -6.06 & -6.15 & -6.07 \\
\hline $\mathrm{H}$ & -2.91 & -2.92 & -2.88 & -2.87 & -2.94 & -2.89 \\
\hline $\mathrm{CH}$ & -6.85 & -7.17 & -7.37 & -7.35 & -7.73 & -7.33 \\
\hline $\mathrm{CH}_{2}$ & -4.47 & -4.64 & -4.66 & -4.65 & $-\mathbf{5 . 0 7}$ & -4.82 \\
\hline $\mathrm{CH}_{3}$ & -2.16 & -2.22 & -2.25 & -2.28 & -2.25 & -2.35 \\
\hline $\mathrm{NH}$ & -5.14 & -5.40 & -5.47 & -5.46 & -5.74 & -5.48 \\
\hline $\mathrm{NH}_{2}$ & -3.04 & -3.13 & -3.49 & -3.50 & -3.50 & -3.55 \\
\hline $\mathrm{NH}_{3}$ & -0.75 & -0.76 & -0.93 & -0.85 & -0.86 & -0.90 \\
\hline $\mathrm{OH}$ & -3.34 & -3.41 & -3.76 & -3.80 & -3.78 & -3.84 \\
\hline $\mathrm{CO}$ & -1.89 & -2.01 & -2.07 & -1.99 & -2.20 & -2.05 \\
\hline $\mathrm{NO}$ & -2.67 & -2.91 & -2.85 & -2.82 & -2.96 & -2.89 \\
\hline $\mathrm{N}_{2}$ & -0.61 & -0.70 & -0.94 & -0.94 & -1.34 & -0.96 \\
\hline
\end{tabular}


Table S5. Preferred binding sites of atomic and molecular species on all model surfaces studied. The site $\mathrm{b}-\mathrm{h}-\mathrm{b}$ denotes a $\mathrm{N}_{2}$ molecule adsorbed to the surface with both its nitrogen atoms (bridge sites connected via a hollow site).

\begin{tabular}{|c|c|c|c|c|c|c|}
\hline Species & $h c p-(0001)$ & $f c c-(111)$ & $f c c-(100)$ & cage-(100) & cage-(100) Pd2 & cage-(100) Pd3 \\
\hline $\mathrm{C}$ & Hcp & Hcp & Hollow & Hollow & Hollow over Ru & Hollow \\
\hline $\mathrm{N}$ & Hcp & Hcp & Hollow & Hollow & Hollow over Ru & Hollow \\
\hline $\mathrm{O}$ & Hcp & Hcp & Hollow & Hollow & Hollow over Ru & Hollow \\
\hline $\mathrm{H}$ & Fcc & Fcc & Bridge & Bridge & Hollow over Pd & Bridge \\
\hline $\mathrm{CH}$ & Hcp & Hcp & Hollow & Hollow & Hollow over Ru & Hollow \\
\hline $\mathrm{CH}_{2}$ & Hcp & Hcp & Hollow & Hollow & Hollow over Ru & Hollow \\
\hline $\mathrm{CH}_{3}$ & Fcc & Fcc & Bridge & Bridge & Bridge over Ru & Bridge \\
\hline $\mathrm{NH}^{\mathrm{H}}$ & Hcp & Hcp & Hollow & Hollow & Hollow over Ru & Hollow \\
\hline $\mathrm{NH}_{2}$ & Bridge & Bridge & Bridge & Bridge & Bridge over Ru & Bridge \\
\hline $\mathrm{NH}_{3}$ & Top & Top & Top & Top & Top & Top \\
\hline $\mathrm{OH}$ & Fcc & Fcc & Hollow & Hollow & Hollow over Pd & Hollow \\
\hline $\mathrm{CO}$ & Top & Hcp & Bridge & Bridge & Hollow over Ru & Top \\
\hline $\mathrm{NO}$ & Hcp & Hcp & Bridge & Bridge & Hollow over Ru & Bridge \\
\hline $\mathrm{N}_{2}$ & Top & Top & b-h-b & b-h-b & b-h-b over Ru & b-h-b \\
\hline
\end{tabular}




\section{References}

(1) Jin, M.; Liu, H.; Zhang, H.; Xie, Z.; Liu, J.; Xia, Y. Nano Res. 2011, 4, 83-91.

(2) Kresse, G.; Furthmuller, J. Phys. Rev. B 1996, 54, 11169-11186.

(3) Kresse, G.; Furthmuller, J. Comput. Mater. Sci. 1996, 6, 15-50.

(4) Blochl, P. E. Phys. Rev. B, 1994, 50, 17953-17979.

(5) Kresse, G.; Joubert, D. Phys. Rev. B 1999, 59, 1758-1775.

(6) Perdew, J. P.; Wang, Y. Phys. Rev. B 1992, 45, 13244-13249.

(7) Monkhorst, H. J.; Pack, J. P. Phys. Rev. B 1976, 13, 5188-5192.

(8) Henkelman, G.; Uberuaga, B. P.; Jonsson, H. J. Chem. Phys. 2000, 113, 9901-9904.

(9) Lide, D. R, CRC Handbook of Chemistry and Physics, 85th ed.; CRC Press: Boca Raton, New York, 2004.

(10) Zhang, L.; Roling, L. T.; Wang, X.; Vara, M.; Chi, M.; Liu, J.; Choi, S.-I.; Park, K.;

Herron, J. A.; Xie, Z.; Mavrikakis, M.; Xia, Y. Science 2015, 349, 412-416.

(11) Watanable, S.; Komine, T.; Kai, T.; Shiiki, K. J. Magn. Magn. Mater. 2000, 220, 277-284.

(12) Kobayashi, M.; Kai, T.; Takano, N.; Shiiki, K. J. Phys.: Condens. Matter 1995, 7, $1835-1842$. 